\title{
Morality in the flesh: on the link between bodily self-consciousness, morality and
}

\section{(dis)honest behaviour}

\author{
Marina Scattolin ${ }^{1,2^{*}}$, Maria Serena Panasiti ${ }^{1,3}, \&$ Salvatore Maria Aglioti ${ }^{1,2^{*}}$ \\ ${ }^{1}$ Sapienza University of Rome and CLNS@Sapienza' Italian Institute of Technology, Rome, \\ Italy \\ ${ }^{2}$ Santa Lucia Foundation, IRCCS, Rome, Italy \\ ${ }^{3}$ Department of Psychology Sapienza University of Rome
}

*Correspondent authors:

Salvatore Maria Aglioti, e-mail address: salvatoremaria.aglioti@uniroma1.it Marina Scattolin, e-mail address: marina.scattolin@uniroma1.it

Keywords: body self-consciousness, body ownership, body agency, moral identity, moral behaviour, honesty 


\begin{abstract}
The sense of owning a body (ownership) and controlling its actions (agency) are two main pillars of bodily self-consciousness (BSC). Although studies suggest that BSC signals may influence morality, the direction of such remains unclear. To investigate this issue, we conducted an online study in which 658 participants completed BSC- and morality-related questionnaires, and undertook a task where they could cheat for monetary gain. We found that those with high sense of ownership, and low reward sensitivity, displayed both high moral identity and deception. This seemingly counterintuitive result supports the notion that people who feel highly moral allow themselves to act immorally. Moreover, we found that high agency was associated with increased moral identity in participants with higher levels of moral disengagement and sense of power. That BSC can differently affect moral identity and behaviour may inspire policies of contrast to immorality and dishonesty, based on changes of corporeal awareness.
\end{abstract}




\section{Introduction}

Corporeal awareness, or bodily self-consciousness (BSC) ${ }^{1}$, is based on two main pillars, namely the feeling of having and owning a body (sense of ownership ${ }^{2}$ ), and that of being able to initiate and control said body's movements (sense of agency ${ }^{3}$ ). The notion that bodily signals may impact higher-order psychological functions is at the core of embodied cognition theories ${ }^{4}$. What remains largely unknown is whether, or how, different aspects of BSC can influence morality and moral decision making. The question of whether, and if so how, awareness of body signals may bias our moral decisions towards dishonesty (e.g., by increasing the temptation from personal rewards) or honesty (e.g., by heightening our sense of responsibility) is central to many religious systems where morality typically plays a fundamental role. However, systematic research on whether body ownership and agency two inherently linked yet distinct constructs ${ }^{5}$ - exert different influences on morality is scarce. It is possible, for example, that an increased sense of ownership over the body is associated with higher levels of dishonesty. Indeed, when signals from the body become more prominent, people appear to indulge in different types of self-serving behaviours, such as making more egoistic offers to others during Ultimatum Games ${ }^{6,7}$ or cheating in exchange for rewards ${ }^{8}$. This effect might be driven by the interoceptive component of body ownership which conveys the reward value assigned to certain stimuli and thus may influence consequent decisions ${ }^{8,9}$. In line with this hypothesis, an increase in rewards sensitivity (as signalled by enhanced activity in the nucleus accumbens during reward anticipation) seems to be associated with more frequent dishonest behaviours ${ }^{10}$. Conversely, sense of agency may be correlated positively with morality and honest behaviours. For instance, existing literature on intentional binding shows that implicit agency is increased when performing moral actions ${ }^{11}$ and reduced when performing immoral ones (i.e., inflicting pain upon others) ${ }^{12}$. Interestingly, the effect of agency on morality might be moderated by such variables as moral 
disengagement and sense of power. Moral disengagement refers to different mechanisms through which personal responsibility over immoral deeds is withdrawn and is often associated with higher deception, even at the risk of one's own reputation ${ }^{13}$. Tellingly, moral disengagement is associated with a reduced sense of agency ${ }^{14}$. As such, heightening one's sense of agency may reduce moral disengagement, and thus increase morality. Studies indicate that the perceived sense of power (i.e., the capacity to influence others ${ }^{15}$ ) may be associated with increased sense of agency, as inferred from intentional binding ${ }^{16}$, and to reduced morality as indexed by increased egoistic and unethical behaviours ${ }^{17}$. Importantly, both bodily representations and morality are quintessentially important for the notion of self. Indeed, people perceive that changes in morality-related characteristics alter one's true self more significantly than changes to personality traits, or even memory loss ${ }^{18,19}$. Moral psychology theories posit that self and morality are two systems that become gradually integrated during adolescence ${ }^{20}$, and that the internalization of social norms is developmentally important for promoting prosocial behaviour ${ }^{21}$. These integration and internalization processes result in the development of a moral self (i.e., the tendency and ability to care about one's own and others' ethical conduct) ${ }^{22}$. Previous investigations on whether the moral self can predict actual behaviours has led to contrasting results. Mounting evidence supports the belief that people act in accordance with their moral identity in an effort to confirm their perceived sense of self $f^{23}$, and that behaviours congruent with this selfconcept are more likely to occur when moral values are actively considered (e.g., by being asked to list the Ten Commandments) ${ }^{24}$ or when such values are perceived as central features of the self $\mathrm{f}^{25}$. However, other studies have shown that relying on people's self-assessment as moral agents may lead to inaccurate predictions of their behaviour ${ }^{26}$. Indeed, once a moral or immoral concept of self is made salient, people may employ subsequent behaviours to reduce inconsistencies with, and therefore preserve, their moral image ${ }^{27}$. For instance, moral 
behaviours are more likely to follow negative actions as an attempt to compensate, and thus avoid having to reconsider one's identity - an effect known as moral cleansing ${ }^{28}$. Conversely, once people have proven that they are not immoral, they feel allowed, or licenced, to act less morally ${ }^{25}$

To further explore these ideas, we conducted an online study to collect self-report measures concerning several variables of interest that allow for the investigation of the possible link(s) between components of BSC and morality. Notably, the participants' morality was not only measured using a questionnaire (Moral Identity), but also by employing a behavioural task where, in one of two blocks, lying was associated with a higher monetary reward. In the Spot the Difference Task (STDT), participants were asked to find 10 details differentiating pairs of images and then report how many (and never which) they had identified (see Fig. 1). Two distinct pairs of images were used, each randomly assigned to only one of the two experimental conditions. In the reward (vs. the no reward) condition, each reported difference above 5 implied an additional pay-off of $€ 0.30$. Moreover, those participants who found the highest number of differences received $€ 10$ at the end of data collection. However, the participants were never told that there only existed five differences. This feature was crucial, as the participants could declare finding more differences than actually existed in order to gain more money. As a measure of (im)moral behaviour (hereafter referred to as Lies) we subtracted five (the actual number of differences) from the numbers reported by the participants.

Participants were also able to exceed the given time limit in an attempt to find more differences and thus receive a higher pay-off. The difference between the participants' response time and the time they were given to complete the task is referred to as the Response Time Lie (RT Lie). We can use this measure as an indicator of a more implicit type of (dis)honesty. 
It is worth noting that a different version of STDT was recently used to highlight the neural correlates of cheating and honesty ${ }^{29}$.

In line with general data replicability recommendations ${ }^{30}$, our sample $(\mathrm{N}=658)$ is consistent with an a priori sample size estimation. We specifically designed our STDT for the study of (dis)honest behaviours in online contexts, and we employed it for the main purpose of investigating whether the senses of ownership and agency are related to (im)moral behaviours and the moral identity of individuals.

\section{Results}

\section{Moral Identity}

We analysed moral identity by using two multiple linear regression models. First, we checked whether any demographic variable (i.e., Age, Sex, Education, Subjective Economic Status or SES) was associated with moral identity. Due to education being significantly correlated $(r=$ $.09,95 \%$ confidence interval, or CI $[.01, .16], p=.023$; Table 1$)$, we included it in both models as a covariate.

The effect of sense of ownership on moral identity.

To investigate whether moral identity was predicted by body ownership, as well as if this effect was moderated by sensitivity to rewards, we entered these two variables and their interaction term as fixed factors in a model predicting moral identity. As residuals were significantly non-normal ( $W=0.94, p<.001)$, we computed (and here present) a robust regression. Sense of ownership significantly increased moral identity $(\beta=0.03,95 \% \mathrm{CI}=$ $[0.02,0.04], t(653)=4.46, \mathrm{p}<.001)$, and the interaction between ownership and reward sensitivity was significant $(\beta=-0.06,95 \% \mathrm{CI}=[-0.11,-0.01], t(653)=-2.28, \mathrm{p}=.023$; Fig. 
2). While sense of ownership increases moral identity when reward sensitivity is low ( $\beta=$ $0.05,95 \% \mathrm{CI}=[0.03,0.07], t(653)=4.28, p<.001$; Fig. 2$)$, the slope for high reward sensitivity is not significantly different from $0(\beta=0.02,95 \% \mathrm{CI}=[-0.01,0.04], t(653)=$ $1.18, p=.238$; Fig. 2).

The effect of sense of agency on moral identity.

In the second model, moral identity was the dependent variable while sense of agency, moral disengagement, and sense of power were the independent variables. To analyse whether moral disengagement and/or sense of power could moderate the effect of agency, we entered two interaction terms as fixed factors (sense of agency $\times$ moral disengagement and sense of agency $\times$ sense of power). We performed a robust regression after a Shapiro-Wilk test showed a significant deviation of residuals from normality $(W=0.95, p<.001)$. The robust results show that sense of agency significantly increased moral identity $(\beta=0.12,95 \% \mathrm{CI}=$ $[0.07,0.17], t(651)=4.98, p<.001)$, while moral disengagement was found to reduce it $(\beta=$ $-0.15,95 \% \mathrm{CI}=[-0.21,-0.10], t(651)=-5.64, p<.001)$. The interaction between agency and moral disengagement was significant $(\beta=0.09,95 \% \mathrm{CI}=[0.04,0.15], t(651)=3.41, p<$ .001 ), and indicated that agency positively predicts moral identity only when moral disengagement is high $(\beta=0.17,95 \% \mathrm{CI}=[0.09,0.26], t(651)=3.87, p<.001 ;$ Fig. 3). Also significant was the interaction between agency and sense of power $(\beta=0.07,95 \% \mathrm{CI}=[0.02$, $0.11], t(651)=2.77, p=.006$; Fig. 4$)$, in that an increase of agency predicts higher moral identity when sense of power is high $(\beta=0.17,95 \% \mathrm{CI}=[0.09,0.25], t(651)=4.01, p<$ .001 ; Fig. 4). 


\section{(Im)moral behaviour}

To ensure that the two pairs of images did not differ in terms of how many details the participants reported finding, we first ran a multiple linear mixed effect model. Lies were the predicted variable, while participants' identification number (ID) and the presented pair of images were entered as the random and fixed factor, respectively. This analysis revealed that the specific pair of images did not predict the number of differences reported by participants $(\beta=0.03,95 \% \mathrm{CI}=[-0.03,0.10], t(655.32)=.99, p=.323)$.

We used three additional multiple linear mixed effect models to analyse lies, and thus investigate whether sense of ownership and agency can predict dishonest behaviour, as well as whether other variables acted as moderators. In all of these analyses, participants' ID's were set as random factors, while their ages were included as fixed predictors (as it significantly correlated with the variable Lies; $r=.08,95 \%$ CI $[.001, .15], p=.049$; Table 1 ).

\section{The effect of sense of ownership on (im)moral behaviour.}

In the first of these models, we entered body ownership, experimental condition (no reward coded as 0 , reward coded as 1), reward sensitivity, and all of the interaction terms, as fixed predictors. Since a Shapiro-Wilk test showed that residuals did not follow a normal distribution $(W=0.56, p<.001)$, we entered the same model in a robust mixed regression analysis. Given the similarity of robust mixed regression results and multiple linear mixed effect model, only the latter - which allows post-hoc comparison testing for mixed effects models - will be reported here. Robust results can be found in the Supplementary Information section. We observed that the condition (no reward/reward) increased the number of lies $(\beta=0.15,95 \% \mathrm{CI}=[0.09,0.22], t(652.41)=4.50, p<.001)$. The interaction between ownership, condition, and reward sensitivity is significant $(\beta=-0.09,95 \% \mathrm{CI}=[-$ $0.17,-0.01], t(651.21)=-2.24, p=.025 ;$ Fig. 5). Indeed, in the reward condition, sense of 
ownership increased the number of lies in participants who scored low in reward sensitivity $(\beta=0.02,95 \% \mathrm{CI}=[0.01,0.05], t(1271.73)=2.26, p=.024 ;$ Fig. 5$)$.

\section{The effect of sense of agency on (im)moral behaviour.}

We computed two models so as to examine the possible link between lies and sense of agency. Agency, experimental condition (no reward coded as 0 , reward coded as 1), moral disengagement, and RT Lies were set as independent variables in one of these models. To investigate possible moderations, we included their interaction (agency $\times$ experimental condition $\times$ moral disengagement $\times$ RT lies) in this analysis. A Shapiro-Wilk test showed that the distribution of residuals was significantly non-normal ( $W=0.57, p<.001)$, leading us to perform a robust regression analysis on the same model. The robust results show that the number of lies in the task increased in association with the experimental condition (no reward/reward $)(\beta=0.001,95 \% \mathrm{CI}=[0.00,0.01], t(662.10)=2.46, p=.014)$ and age $(\beta=$ $0.001,95 \% \mathrm{CI}=[0.00,0.01], t(660.90)=3.44, p<.001)$. All other predictors were not significant.

In the last model, sense of agency, condition (no reward; reward), sense of power, RT Lies and all interactions between these (agency $\times$ experimental condition $\times$ sense of power $\times$ RT Lies) were fixed factors. We computed a robust multiple linear mixed effect model due to the significantly non-normal residuals $(W=0.55, p<.001)$. The robust analysis found that the number of reported differences increased in association with age $(\beta=0.001,95 \% \mathrm{CI}=[0.00$, $0.01], t(1064.29)=3.46, p<.001)$, yet no other predictor was significant.

\section{Discussion}

We have investigated whether two of the basic pillars of bodily self-consciousness (the senses of ownership and agency) are differentially related to moral identity and (im)moral 
behaviour. Specifically, we tested if sensitivity to rewards could modulate the effect of body ownership, while concurrently exploring whether moral disengagement and sense of power could impact the effect of the sense of agency. To answer these questions, we conducted an online study where moral identity was measured by means of a questionnaire and (im)moral behaviour was measured by a task that tempted participants to lie in exchange for a higher monetary reward.

Interestingly, we found that both sense of ownership and sense of agency strengthened moral identity. The relation between moral identity and sense of agency may possibly be explained through personal responsibility. In fact, sense of agency over the body entails feelings of responsibility over the outcomes of performed actions ${ }^{11}$. Feeling responsible for the effects of one's own behaviours may thus promote the occurrence of moral deeds ${ }^{31}$ and, by consequence, consolidate one's moral concept of the self. In line with this, we found that acting dishonestly was associated with decreased readiness potential - an electrocortical signal associated with the production of voluntary acts and strongly associated with a sense of responsibility ${ }^{32}$. While the link between sense of agency and moral identity could well be rooted in feelings of responsibility, the positive relation between sense of ownership and moral identity appears to be less straightforward. However, this association may resemble that found between enhanced body ownership and positive attributes. Consider, for example, the enfacement illusion ${ }^{33}$, i.e., the inclusion of another person's face into an extended representation of one's own body. Crucially, this extension of the sense of body ownership is more likely to occur when the other person is considered as displaying desirable characteristics, such as niceness and physical attractiveness ${ }^{34,35}$. Therefore, it has been proposed that enhanced body ownership is observed in association with positive qualities, as doing so promotes the observer to attribute these qualities to themselves ${ }^{36}$. The positive relation we found between sense of ownership and moral identity may be aligned to this, as 
increased ownership could facilitate the association between body and positive characteristics (like morality), that are consequently ascribed to the self.

Notably, we found that other variables modulate the relation between each specific component of BSC and morality. In particular, reward sensitivity appears to moderate the main effect of sense of ownership. Moreover, we observed this both for moral identity and immoral behaviour. In fact, an important result of our study is that awareness of one's own body can enhance moral self-image, which in turn may steer one towards dishonesty. Indeed, we found that, as the sense of ownership intensifies, low levels of reward sensitivity become associated with increased moral identity (Fig. 2) and, concurrently, to increased deceptive behaviour during the STDT (Fig. 5). This (seemingly paradoxical) result may be in keeping with previous studies which indicate that, when people perceive themselves as highly moral, they feel more licenced to act less morally - an effect referred to as moral licencing ${ }^{25,27}$. Interestingly, we observed no such pattern for high levels of reward sensitivity. Indeed, the above effect tended to occur when an enhanced sense of body ownership conveyed signals of low reward sensitivity. Our results suggest that being less sensitive to reward may have a paradoxical effect on reward-related behaviours. This supports the work of Capa and Bouquet $^{37}$, who showed that participants with low sensitivity to reward perform better in working memory tasks when performance is associated with higher rewards, and when such benefits are presented subliminally. However, other implicit measures of reward sensitivity suggest a different association with (im)moral behaviour. In a recent study from our group ${ }^{38}$ we asked participants to play a game in which they were tempted to lie to people associated with different social characteristics for a monetary reward ${ }^{39}$. Before the (im)moral choice was made, we measured participants' tendency to look at the game outcome before the information regarding their opponent's social status. This served as an implicit measure of 
reward sensitivity: the weaker this measure was, the less participants behaved immorally towards low-status opponents ${ }^{38}$.

Traditionally speaking, the way in which reward sensitivity influences reward-related behaviours (e.g., addiction) has been believed to be non-linear ${ }^{40}$. According to the Reward Deficiency $\mathrm{Model}^{41}$, low sensitivity to reward encourages people to seek bigger rewards in order to boost a depressed dopamine system. Concurrently, however, similar reward-seeking behaviours are observed in association with hypersensitivity to rewards ${ }^{42,43}$.

Another novel result is that a higher sense of agency seems to strengthen moral identity in people who employ justification strategies for their wrongdoings. This suggests that feeling in control of one's own actions can partly counterbalance the negative effect of moral disengagement. Indeed, Bandura and colleagues ${ }^{31}$ argued that, when people are faced with multiple, alternative courses of action, they aim at behaving in accordance with their moral standards. To achieve their goals while simultaneously avoiding immoral behaviours, they can employ self-censure mechanisms when making decisions. Self-sanctions appear to be strongly activated when the sense of responsibility over one's own actions and outcomes is high, which is to say when justifying future detrimental behaviour becomes difficult. Accordingly, our data suggest that sense of agency does increase moral identity, especially when moral disengagement is high. While sense of agency and moral disengagement appear to predict moral identity, their relation to behaviour in a given task does not appear to be significant. One possible explanation for this is that anticipatory self-sanction mechanisms were activated once participants were presented with the opportunity of cheating in exchange for a higher pay-off. Therefore, self-sanctions may have prevented the use of moral disengagement strategies and consequently restrained deceptive behaviours. Contrary to what we expected, agency boosted the effect of sense of power over moral identity. Simply put, sense of agency was associated with higher moral identity in 
participants who reported being able to influence others. This finding supports previous research, which has shown that feeling powerful is associated with a preference for deontological moral judgements ${ }^{44}$, which may in turn enforce a moral concept of self. However, our study shows a lack of association between (im)moral behaviour, sense of agency, and sense of power. This may be explained by the complex pattern of relations among these and other variables, as well as by the link between sense of power and opposite moral-thinking styles. A recent study ${ }^{45}$ found that sense of power is associated to different styles of moral thinking, namely integration-oriented (which relies on affective and cognitive evaluations), deliberation-oriented (where the focus of the decision-making process is to maximize outcomes), and rule-oriented (focused on abiding by existing rules). Notably, the study suggests that these moral orientations may have opposite effects over moral judgements. This means that, while deliberation- and integration-oriented styles seem to favour outcome-based judgements, rule-orientation styles act in the opposite direction. Furthermore, deontological judgements seem to increase in association with integrationoriented styles, and are reduced by deliberation-oriented moral thinking. In light of all this, we can suggest that, during our task, the participants' sense of power may have activated different moral-thinking styles. It is possible that their opposing effects may have counteracted each other's bias towards (dis)honest behaviours, ultimately leading to the absence of any relation between the senses of agency and power.

While it is our belief that the present study significantly expands the current knowledge on the role of body awareness into higher level cognitive functions, such as morality and moral decision-making, we acknowledge some limitations. Our participants always completed the task before being asked to respond to the questionnaire assessing moral identity. We made this choice so as to avoid the activation of any self-related concept of morality prior to performing the task, which might have influenced spontaneous behaviour. Mazar and 
colleagues ${ }^{24}$ showed that cheating did not change participants' opinion regarding their honesty. As such, we believe that performing the task did not bias responses in the moral identity questionnaire. However, it could be argued that, as this questionnaire always followed the STDT, our results may reflect personal efforts to restore moral self-image by a figurative act of cleansing ${ }^{27,28}$. Indeed, people often employ various strategies to try and dissociate themselves from immoral deeds ${ }^{14,46}$. Dissociating immorality from the self prevents having to review one's own moral image in order to comprehend inconsistent behaviours and preserve one's moral reputation in the eyes of others ${ }^{26}$. In this vein, it is possible that participants who lied more in our task may have been attempting to re-establish their concept of self by overestimating just how importantly they consider certain moral characteristics. Accordingly, studies indicate that those who are less interested in upholding their moral image may present themselves more truthfully in spite of immoral behaviours 26,47 , while the desire to maintain a moral facade may paradoxically lead to increased dishonesty or, in this case, to an inflated moral self ${ }^{26}$. Although we tested a large sample of people, all of them were Italian-speaking, thereby complicating the generalization of the results. The very cogent issue of whether culture can influence the way in which body ownership and agency - as well as moderating variables impact morality and (dis)honest behaviour ought be addressed in future studies. In particular, research should clarify whether cross-cultural differences in morality ${ }^{48}$ and moral behaviours ${ }^{49}$ could be traced to possible differences in awareness of bodily signals ${ }^{50}$, or in the feelings of agency associated with pleasant or desirable outcomes ${ }^{51}$. In conclusion, we have demonstrated that different aspects of morality can be accounted for by different components of BSC. In fact, we observed increased moral identity in association with enhanced senses of ownership and agency. However, different variables appear to intervene in these relations: while the effect of sense of agency appears modulated by moral 
disengagement and sense of power, reward sensitivity moderated the effect of sense of ownership. When considering dishonest behaviours, our data suggest that these are predicted by increased sense of ownership, but only when reward sensitivity is low. Policies aimed at increasing corporeal awareness may strengthen the moral identity of individuals. At the same time, understanding which factors operate in synergy with BSC components during decisionmaking processes could help develop specific training programmes. These would aid in promoting a reliance on mechanisms that could counterbalance the tendency to act immorally.

\section{Methods}

We pre-registered our methods and analysis plan on the Open Science Framework (see https://osf.io/scbnw).

We used RStudio, version $1.3 .959^{52}$, to analyse the data: correlations and normality of residuals were computed using functions cor.test and shapiro.test of the stats package (version 4.0.2 $2^{53}$, respectively. To perform the multiple linear regression and generalized linear mixed effect models regression analysis, we used package lme 4 version $1.1-23^{54}$, which was also employed to determine effect sizes. Robust linear regressions were computed using package MASS, version 7.3 - 51.6 $6^{55}$, while robust linear mixed effect models were performed using package robustlmm, version $2.3^{56}$. All analyses were two-tailed.

\section{Participants}

We recruited our participants via Prolific (http://www.prolific.co), and they took part in the study from December 2018 to February 2019 - before the widespread effects of the COVID19 pandemic could bias their responses. Sample size was determined following two a priori power analyses for General Linear Models, which we performed via R software ( $p w r$ 
package) with $\alpha=.05$, a small effect size (.02) and power of 0.8 . The results indicate that 550 and 647 responses were needed for 3 and 5 predictors, respectively.

A total of 705 participants took part in the study, 47 of whom were excluded from the final dataset (the full data for both reasons and occurrences are reported in Supplementary Table 1). Our final sample included 658 participants (females $=314)$ between the ages of $18-66(\mathrm{M}$ $=29.37, \mathrm{SD}=9.68$ ), who declared compliance with the requirements of the study (i.e., not having any neurological or psychiatric condition, nor taking any psychiatric drugs). Italian was the first language of all the participants. All of the participants gave their informed consent and were paid for their participation. The methods and procedures of this study were approved by the Ethic Committee of the IRCCS Fondazione Santa Lucia (Rome), and were in accordance with the Declaration of Helsinki.

\section{Materials}

We asked the participants to complete a series of questionnaires measuring different components of BSC, with the intent of assessing their possible link with moral identity and behaviour, as well as exploring which other variables could modulate this relation. All questionnaires, and the online behavioural task, were developed using PsyToolkit (version 2.5.4 $)^{57}$. Additional information on the measures used in the study can be found in the Supplementary Information.

\section{Morality measures}

Moral identity. We assessed the Moral identity of participants using the internalization factor of the Moral Identity Measure ${ }^{58}$ (e.g., 'Being someone who has these characteristics is an important part of who I am'; $\alpha=.79$ ). 
(Im)moral behaviour. To assess the (im)moral behaviour of our participants, we used a version of the STDT in which participants were presented with pairs of images in the middle of the screen, and asked to find as many differences as possible within 45 seconds. A countdown was displayed on the top centre of the screen while numbers from 0 to 10 were shown on the bottom. Once the countdown had ended, the participants were then instructed to click on the number corresponding to the differences they had found. The participants were informed that 10 differences existed between the image pairs and that, given the same amount of time, most people found only 5 of these. All participants completed the task twice under two different conditions (no reward and reward), the order of which was randomized. Each condition was completed with one of two possible image pairs. We used a web search to obtain these, and other, images. We based the selection of the two image pairs employed in the STDT on a validation study (see the Supplementary Information section for a full description). For every participant, each image pair was randomly assigned to only one experimental condition.

In the no reward condition, participants gained $€ 0.10$ for completing the task; in the reward condition, further to the aforementioned $€ 0.10$, participants received an additional $€ 0.30$ for each difference above 5 they reported finding, plus a further $€ 10$ if, at the end of data collection, they were among those who found the highest number of differences. Crucially, the images in each pair only differed in five details, so any response above this number was considered a lie. Lies were calculated by subtracting the actual number of differences from the responses (results smaller than, or equal to, zero were coded as zero). Participants also had the opportunity to enter their responses after the countdown had ended. Indeed, both the images and response options continued to be displayed after the countdown. Any response given after 45s was considered an RT Lie. We computed RT Lies with a similar calculation 
to that of lies: the time participants were given to complete the task (45s) was subtracted from their response time (results smaller than, or equal to, zero were coded as zero).

By way of example, and due to copyright regulations, Fig. 1 shows a mock pair of images specifically created by the authors for the purpose of publication.

\section{Body self-consciousness measures}

We used two scales to measure sense of ownership, namely the private body subscale of the Body Consciousness Questionnaire ${ }^{59}$ (e.g., 'I am sensitive to internal bodily tensions'; $\alpha=$ $.65)$ and the ownership subscale of the Embodied Sense of Self Scale ${ }^{60}$ (e.g., 'Sometimes the clothes I am wearing feel heavy', $\alpha=.73$ ). As stated in the pre-registration, participants' responses to the two ownership questionnaires were to be combined into a single measure after reversing responses to the ESSS. In fact, while the BCQ measures interoceptive awareness, higher ESSS scores reflect a more anomalous sense of ownership, such as that observed in schizophrenia ${ }^{60}$. Although we expected these two measures to be negatively correlated, their Pearson's correlation coefficient was positive $(r=.16,95 \%$ CI $[.09, .24], p<$ .001 ; see Table 1), which indicated that the two could not be combined. Previous studies have suggested that interoception modulates the degree to which a virtual hand or body is perceived as belonging to the self ${ }^{61-64}$, thereby supporting the notion that - although not sufficient - inner body signals are necessary for sense of ownership ${ }^{65}$. Consequently, we only used BCQ scores in those models with body ownership as a predictor.

For sense of agency, we employed the positive agency subscale from Tapal and colleagues ${ }^{66}$ (e.g., 'I am in full control of what I do'). This showed positive internal consistency $(\alpha=.78)$. 


\section{Moderating variables}

Considering that the task included a financial reward, we used the Monetary Intelligence Scale $^{67}$ as a measure of reward sensitivity, which consists of the Affective $(\alpha>.85)$, Behavioural $(\alpha>$.7), and Cognitive $(\alpha>$.85) sub-constructs. The 8-item Propensity to Morally Disengage Scale ${ }^{68}(\alpha=.77)$ and Sense of Power Scale ${ }^{15}(\alpha=.85)$ were used to assess moral disengagement and sense of power, respectively.

\section{Procedure}

The participants could complete all parts of the survey using any computer with an internet connection. We also provided them with general information regarding the study. In the consent page, participants had to check a box to indicate their agreement to participation not doing so would bar them from completing the subsequent steps. Demographic information (i.e., age, sex, highest education degree received, subjective economic status) was collected prior to the STDT. To assess subjective economic status (SES), we asked the participants to place themselves along a Visual Analogue Scale, ranging from 'People who have the least money' to 'People who have the most money' (scores ranged from 0 to 100). This method was adapted from Adler et al. ${ }^{69}$ and Ostrove et al. ${ }^{70}$.

Questionnaires followed the task. The order of conditions and pairs of images in the STDT was randomized, as was the order of questionnaires and the questions themselves. The Monetary Intelligence Scale and Sense of Agency Scale included one attention-check question each, namely 'answer "agree" to this sentence' and 'the answer to this question should be "disagree"". The entire study could be completed in approximately 20 minutes. 


\section{Statements}

\section{Data Availability}

Part of the participants $(\mathrm{N}=32,4.86 \%$ of the final sample $)$ who took part to this study did not consent to the re-use of their data in new studies. For this reason, data of these participants will only be included in a dataset for replication of all analyses presented here. Once this manuscript is published in a peer-reviewed journal, the dataset will be made available to anyone upon personal communication with the authors of this study and only upon agreeing to use the dataset exclusively for replication purposes. The data of those participants who consented to re-use will be made available to anyone who wishes to test new hypotheses. A DOI will be created at the moment of publication in a peer-reviewed journal.

\section{Code Availability}

The code for replication of analyses presented here will be available at the moment of publication of this manuscript in a peer-reviewed journal.

\section{Acknowledgements}

The present work was funded by a European Research Council (ERC) Advanced Grant 2017 (Embodied Honesty in real world and digital interactions - eHONESTY) (grant awarded to SMA, grant number ERC-2017-AdG - eHONESTY - 789058).

\section{Competing Interests}

All co-authors of this paper have no financial or other conflicts of interest 


\section{Reference}

1. Berlucchi, G. \& Aglioti, S. M. The body in the brain: neural bases of corporeal awareness. Trends in Neurosciences 20, 560-564 (1997).

2. Blanke, O. Multisensory brain mechanisms of bodily self-consciousness. Nature Reviews Neuroscience 13, 556-571 (2012).

3. Haggard, P. Sense of agency in the human brain. Nature Reviews Neuroscience 18, 196-207 (2017).

4. Barsalou, L. W. Grounded cognition. Annu. Rev. Psychol 59, 617-645 (2008).

5. Tsakiris, M., Longo, M. R. \& Haggard, P. Having a body versus moving your body: Neural signatures of agency and body-ownership. Neuropsychologia 48, 2740-2749 (2010).

6. Mancini, A. et al. Suffering makes you egoist: Acute pain increases acceptance rates and reduces fairness during a bilateral ultimatum game. PLoS One 6, e26008 (2011).

7. Lenggenhager, B., Azevedo, R. T., Mancini, A. \& Aglioti, S. M. Listening to your heart and feeling yourself: Effects of exposure to interoceptive signals during the ultimatum game. Exp Brain Res 230, 233-241 (2013).

8. Williams, E. F., Pizarro, D., Ariely, D. \& Weinberg, J. D. The Valjean effect: Visceral states and cheating. Emotion 16, 897-902 (2016).

9. Paulus, M. P. Decision-making dysfunctions in psychiatry--altered homeostatic processing? Science 318, 602-606 (2007).

10. Abe, N. \& Greene, J. D. Response to anticipated reward in the nucleus accumbens predicts behavior in an independent test of honesty. The Journal of Neuroscience $\mathbf{3 4}$, 10564-10572 (2014).

11. Moretto, G., Walsh, E. \& Haggard, P. Experience of agency and sense of responsibility. Consciousness and Cognition 20, 1847-1854 (2011). 
12. Caspar, E. A., Cleeremans, A. \& Haggard, P. Only giving orders? An experimental study of the sense of agency when giving or receiving commands. PLoS One 13, e0204027 (2018).

13. Panasiti, M. S., Pavone, E. F., Merla, A. \& Aglioti, S. M. Situational and dispositional determinants of intentional deceiving. PLoS ONE 6, e19465 (2011).

14. Bandura, A. Social cognitive theory: An agentic perspective. Annu. Rev. Psychol 52, $1-26(2001)$.

15. Anderson, C., John, O. P. \& Keltner, D. The personal sense of power. Journal of Personality 80, 313-344 (2012).

16. Obhi, S. S., Swiderski, K. M. \& Brubacher, S. P. Induced power changes the sense of agency. Consciousness and Cognition 21, 1547-1550 (2012).

17. Lammers, J., Galinsky, A. D., Dubois, D. \& Rucker, D. D. Power and morality. Current Opinion in Psychology 6, 15-19 (2015).

18. Strohminger, N., Knobe, J. \& Newman, G. The true self: A psychological concept distinct from the self. Perspectives on Psychological Science 12, 551-560 (2017).

19. Strohminger, N. \& Nichols, S. The essential moral self. Cognition 131, 159-171 (2014).

20. Bergman, R. Why Be Moral? A Conceptual Model from Developmental Psychology. Human Development 45, 104-124 (2002).

21. Davidov, M., Vaish, A., Knafo-Noam, A. \& Hastings, P. D. The motivational foundations of prosocial behavior from a developmental perspective-Evolutionary roots and key psychological mechanisms: Introduction to the special section. Child Development 87, 1655-1667 (2016).

22. Krettenauer, T. The dual moral self: Moral centrality and internal moral motivation. The Journal of Genetic Psychology 172, 309-328 (2011). 
23. Stets, J. E. \& Carter, M. J. The moral self: Applying identity theory. Social Psychology Quarterly 74, 192-215 (2011).

24. Mazar, N., Amir, O. \& Ariely, D. The dishonesty of honest people: A theory of selfconcept maintenance. Journal of Marketing Research 45, 633-644 (2008).

25. Effron, D. A. \& Conway, P. When virtue leads to villainy: Advances in research on moral self-licensing. Current Opinion in Psychology 6, 32-35 (2015).

26. Ellemers, N., van der Toorn, J., Paunov, Y. \& van Leeuwen, T. The psychology of morality: A review and analysis of empirical studies published from 1940 through 2017. Personality and Social Psychology Review 23, 332-366 (2019).

27. Sachdeva, S., Iliev, R. \& Medin, D. L. Sinning saints and saintly sinners. The paradox of moral self-regulation. Psychological Science 20, 523-528 (2009).

28. West, C. \& Zhong, C.-B. Moral cleansing. Current Opinion in Psychology vol. 6 $221-225$ (2015).

29. Speer, S. P. H., Smidts, A. \& Boksem, M. A. S. Cognitive control increases honesty in cheaters but cheating in those who are honest. Proceedings of the National Academy of Sciences of the United States of America 117, 19080-19091 (2020).

30. Asendorpf, J. B. et al. Recommendations for increasing replicability in psychology. European Journal of Personality 27, 108-119 (2013).

31. Bandura, A., Barbaranelli, C., Caprara, G. V. \& Pastorelli, C. Mechanisms of moral disengagement in the exercise of moral agency. Journal of Personality and Social Psychology 71, 364-374 (1996).

32. Panasiti, M. S. et al. The motor cost of telling lies: Electrocortical signatures and personality foundations of spontaneous deception. Social Neuroscience 9, 573-589 (2014). 
33. Porciello, G., Bufalari, I., Minio-Paluello, I., di Pace, E. \& Aglioti, S. M. The "Enfacement" illusion: A window on the plasticity of the self. Cortex 104, 261-275 (2018).

34. Bufalari, I., Lenggenhager, B., Porciello, G., Holmes, B. S. \& Aglioti, S. M. Enfacing others but only if they are nice to you. Frontiers in Behavioral Neuroscience 8, 102 (2014).

35. Sforza, A., Bufalari, I., Haggard, P. \& Aglioti, S. M. My face in yours: Visuo-tactile facial stimulation influences sense of identity. Social Neuroscience 5, 148-162 (2010).

36. Scattolin, M., Panasiti, M. S. \& Aglioti, S. M. Body ownership as a proxy for individual and social separation and connection. Behavioral and Brain Sciences (accepted, 2021).

37. Capa, R. L. \& Bouquet, C. A. Individual differences in reward sensitivity modulate the distinctive effects of conscious and unconscious rewards on executive performance. Frontiers in Psychology 9, 148 (2018).

38. Schepisi, M., Porciello, G., Aglioti, S. M. \& Panasiti, M. S. Oculomotor behavior tracks the effect of ideological priming on deception. Scientific Reports 10, 9555 (2020).

39. Azevedo, R. T., Panasiti, M. S., Maglio, R. \& Aglioti, S. M. Perceived warmth and competence of others shape voluntary deceptive behaviour in a morally relevant setting. British Journal of Psychology 109, 25-44 (2017).

40. Davis, C. \& Fox, J. Sensitivity to reward and body mass index (BMI): Evidence for a non-linear relationship. Appetite 50, 43-49 (2008). 
41. Blum, K. et al. The reward deficiency syndrome: A biogenetic model for the diagnosis and treatment of impulsive, addictive and compulsive behaviors. Journal of Psychoactive Drugs 32, 1-112 (2000).

42. Davis, C. et al. From motivation to behaviour: A model of reward sensitivity, overeating, and food preferences in the risk profile for obesity. Appetite 48, 12-19 (2007).

43. Ponsi, G., Scattolin, M., Villa, R. \& Aglioti, S. M. Human moral decision-making through the lens of Parkinson's disease. NPJ Parkinson's Dis. (accepted, 2021)

44. Lammers, J. \& Stapel, D. A. How power influences moral thinking. Journal of Personality and Social Psychology 97, 279-289 (2009).

45. Fleischmann, A., Lammers, J., Conway, P. \& Galinsky, A. D. Paradoxical Effects of Power on Moral Thinking: Why Power Both Increases and Decreases Deontological and Utilitarian Moral Decisions. Social Psychological and Personality Science 10, 110-120 (2019).

46. Zhong, C.-B. \& Liljenquist, K. Washing away your sins: Threatened morality and physical cleansing. Science 313, 1451-1452 (2006).

47. Halevy, R., Shalvi, S. \& Verschuere, B. Being Honest About Dishonesty: Correlating Self-Reports and Actual Lying. Human Communication Research 40, 54-72 (2014).

48. Stankov, L. \& Lee, J. Nastiness, Morality and Religiosity in 33 nations. Personality and Individual Differences 99, 56-66 (2016).

49. Graham, J., Meindl, P., Beall, E., Johnson, K. M. \& Zhang, L. Cultural differences in moral judgment and behavior, across and within societies. Current Opinion in Psychology 8, 125-130 (2016). 
50. Ma-Kellams, C. Cross-cultural differences in somatic awareness and interoceptive accuracy: A review of the literature and directions for future research. Frontiers in Psychology 5, 1379 (2014).

51. Barlas, Z. \& Obhi, S. S. Cultural background influences implicit but not explicit sense of agency for the production of musical tones. Consciousness and Cognition 28, 94 103 (2014).

52. RStudio Team. RStudio: Integrated Development Environment for R. (2020).

53. R Core Team. R: A language and environment for statistical computing. (2020).

54. Bates, D., Mächler, M., Bolker, B. \& Walker, S. Fitting Linear Mixed-Effects Models Using \{lme4\}. Journal of Statistical Software 67, 1-48 (2015).

55. Venables, W. N. \& Ripley, B. D. Modern Applied Statistics with S. (Springer, 2002). doi:https://doi.org/10.1007/978-0-387-21706-2.

56. Koller, M. Robustlmm: An R package for Robust estimation of linear Mixed-Effects models. Journal of Statistical Software 75, 1-24 (2016).

57. Stoet, G. PsyToolkit: A novel web-based method for running online questionnaires and reaction-time experiments. Teaching of Psychology 44, 24-31 (2017).

58. Aquino, K. \& Reed, A. The self-importance of moral identity. Journal of Personality and Social Psychology 83, 1423-1440 (2002).

59. Miller, L., Murphy, R. \& Buss, A. H. Consciousness of body: Private and public. Journal of Personality and Social Psychology 41, 397-406 (1981).

60. Asai, T., Kanayama, N., Imaizumi, S., Koyama, S. \& Kaganoi, S. Development of embodied sense of self scale (ESSS): Exploring everyday experiences induced by anomalous self-representation. Frontiers in Psychology 7, 1005 (2016). 
61. Aspell, J. E. et al. Turning body and self inside out: Visualized heartbeats alter bodily self-consciousness and tactile perception. Psychological Science 24, 2445-2453 (2013).

62. Suzuki, K., Garfinkel, S. N., Critchley, H. D. \& Seth, A. K. Multisensory integration across exteroceptive and interoceptive domains modulates self-experience in the rubber-hand illusion. Neuropsychologia 51, 2909-2917 (2013).

63. Monti, A., Porciello, G., Tieri, G. \& Aglioti, S. M. The "embreathment” illusion highlights the role of breathing in corporeal awareness. Journal of Neurophysiology 123, 420-427 (2020).

64. Heydrich, L. et al. Cardio-visual full body illusion alters bodily self-consciousness and tactile processing in somatosensory cortex. Scientific Reports 8, 9230 (2018).

65. Blanke, O., Slater, M. \& Serino, A. Behavioral, Neural, and Computational Principles of Bodily Self-Consciousness. Neuron vol. 88 145-166 (2015).

66. Tapal, A., Oren, E., Dar, R. \& Eitam, B. The sense of agency scale: A measure of consciously perceived control over one's mind, body, and the immediate environment. Frontiers in Psychology 8, 1552 (2017).

67. Tang, T. L.-P. et al. Monetary intelligence and behavioral economics across 32 cultures: Good apples enjoy good quality of life in good barrels. J Bus Ethics 148, 893-917 (2018).

68. Moore, C., Detert, J. R., Trevino, L. K., Backer, V. L. \& Mayer, D. M. Why employees do bad things: Moral disengament and unethical organizational behavior. Personnel Psychology 65, 1-48 (2012).

69. Adler, N. E., Epel, E. S., Castellazzo, G. \& Ickovics, J. R. Relationship of subjective and objective social status with psychological and physiological functioning: preliminary data in healthy, white women. Health Psychology 19, 586-592 (2000). 
70. Ostrove, J. M., Adler, N. E., Kuppermann, M. \& Washington, A. E. Objective and subjective assessments of socioeconomic status and their relationship to self-rated health in an ethnically diverse sample of pregnant women. Health Psychology 19, 613-618 (2000). 


\section{Tables}

\begin{tabular}{|c|c|c|c|c|c|c|}
\hline Variable & 1 & 2 & 3 & 4 & 5 & 6 \\
\hline 1. Age & & & & & & \\
\hline 2. Education & $\begin{array}{l}.25 * * * \\
{[.17, .32]}\end{array}$ & & & & & \\
\hline 3. SES & $\begin{array}{l}-.17 * * * \\
{[-.24,-.09]}\end{array}$ & $\begin{array}{l}.13 * * * \\
{[.06, .21]}\end{array}$ & & & & \\
\hline 4. BCQ & $\begin{array}{c}.07 \\
{[-.01, .14]}\end{array}$ & $\begin{array}{l}-.02 \\
{[-.10, .05]}\end{array}$ & $\begin{array}{l}-.09 * \\
{[-.17,-.01]}\end{array}$ & & & \\
\hline 5. ESSS & $\begin{array}{l}-.04 \\
{[-.11, .04]}\end{array}$ & $\begin{array}{l}-.06 \\
{[-.14, .01]}\end{array}$ & $\begin{array}{l}-.09 * \\
{[-.16,-.01]}\end{array}$ & $\begin{array}{l}.16 * * * \\
{[.09, .24]}\end{array}$ & & \\
\hline $\begin{array}{l}\text { 6. Moral } \\
\text { Identity }\end{array}$ & .03 & $.09 *$ & .00 & $.15^{* * *}$ & $-.08 *$ & \\
\hline & {$[-.05, .11]$} & {$[.01, .16]$} & {$[-.07, .08]$} & {$[.08, .23]$} & {$[-.16,-.01]$} & \\
\hline 7. Lies & $\begin{array}{l}.08 * \\
{[.001, .15]}\end{array}$ & $\begin{array}{l}-.03 \\
{[-.11, .04]}\end{array}$ & $\begin{array}{l}-.01 \\
{[-.09, .07]}\end{array}$ & $\begin{array}{l}.03 \\
{[-.05, .10]}\end{array}$ & $\begin{array}{l}.09 * \\
{[.01, .17]}\end{array}$ & $\begin{array}{l}-.10 * \\
{[-.17,-.02]}\end{array}$ \\
\hline
\end{tabular}

\section{Table 1.}

Table reporting Pearson's correlation coefficients $(r)$ between demographic variables (Age, Education, Subjective Economic Status or SES), two ownership questionnaires (Body Consciousness Questionnaire or BCQ, Embodied Sense of Self Scale or ESSS), and two morality measures (Moral Identity and Lies in the Spot The Difference Task). Values in square brackets represent $95 \%$ confidence intervals. Asterisks indicate significance $(* p<$ $.05, * * p<.01, * * * p<.001)$. 
Figures



Fig. 1. Example of Spot The Difference Task (STDT) trial. The task was composed of two trials, which were completed by all participants under two different conditions - no reward and reward. In both of the STDT trials, participants were asked to compare a pair of images in order to find as many differences as they could in 45 seconds. Participants were told that the images contained 10 details, yet the majority of people could only find 5 in the given timeframe. In the reward condition (vs. no reward condition) participants obtained $€ 0.30$ for each difference above 5 they reported finding. At the end of the data collection process, the participants who identified the highest number of differences received an additional $€ 10$. Crucially, and unbeknownst to the participants, only five differences existed between the images. Any response above five could then be considered a Lie. A countdown signalling the 
remaining time (in seconds) was displayed on the top-centre of the screen. At any time, participants could indicate the number of identified differences by clicking on the corresponding number within the yellow dots. Crucially, once the countdown hit zero, the participants could still compare the two images, as these remained on-screen until a response was provided. The dashed line represents a possible delayed response. The additional time that participants took to find differences and provide a response was used as a measure of a more implicit type of dishonesty (RT Lie). Due to copyright regulations, the above figure shows a mock pair of images not shown during the study. The images presented here were created by the authors for the sole purpose of exemplifying the STDT. 




Fig. 2. Moral identity scores as a function of ownership and reward sensitivity. The plot shows regression lines for 1SD below (Low Reward Sensitivity) and above (High Reward Sensitivity) the mean reward sensitivity score. The shaded bands represent $95 \%$ confidence intervals, and the asterisks indicate significance $(* * * p<.001)$. 




Fig. 3. Moral identity scores as a function of agency and moral disengagement. The plot shows regression lines for 1SD below (Low Moral Disengagement) and above (High Moral Disengagement) the mean moral disengagement score. The shaded bands represent $95 \%$ confidence intervals and the asterisks indicate significance $(* * * p<.001)$. 




Fig. 4. Moral identity scores as a function of agency and sense of power. The plot shows regression lines for 1SD below (Low Sense of Power) and above (High Sense of Power) the mean sense of power score. The shaded bands represent $95 \%$ confidence intervals and the asterisks indicate significance $(* * * p<.001)$. 
a

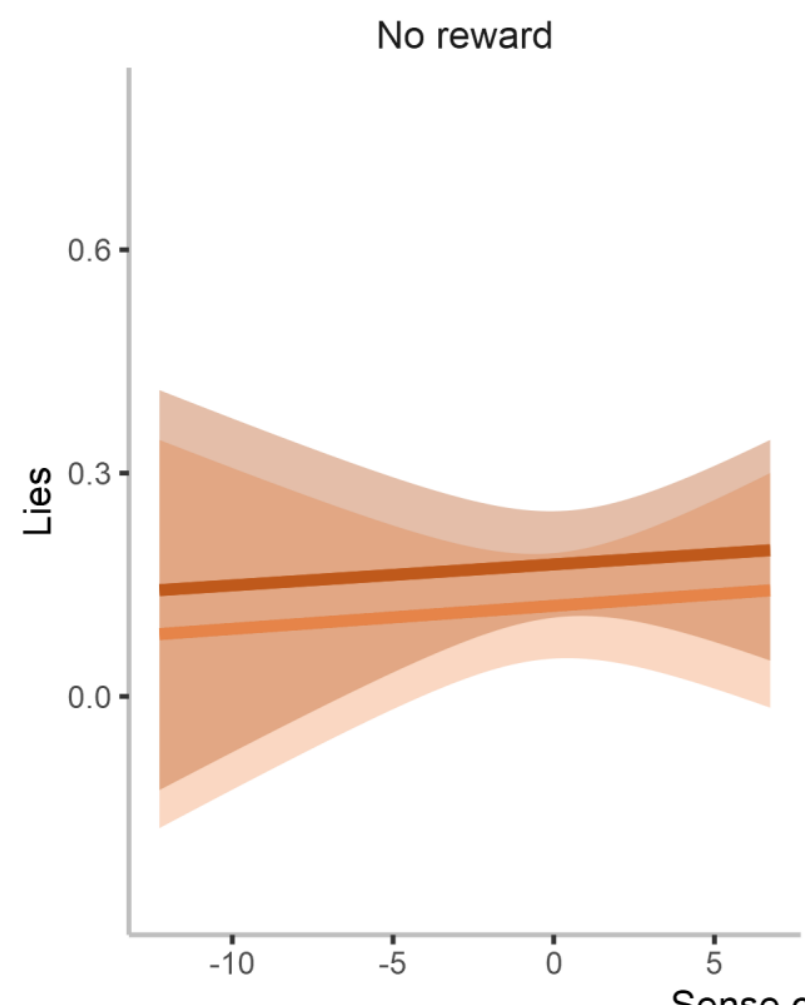

Sense of ownership b

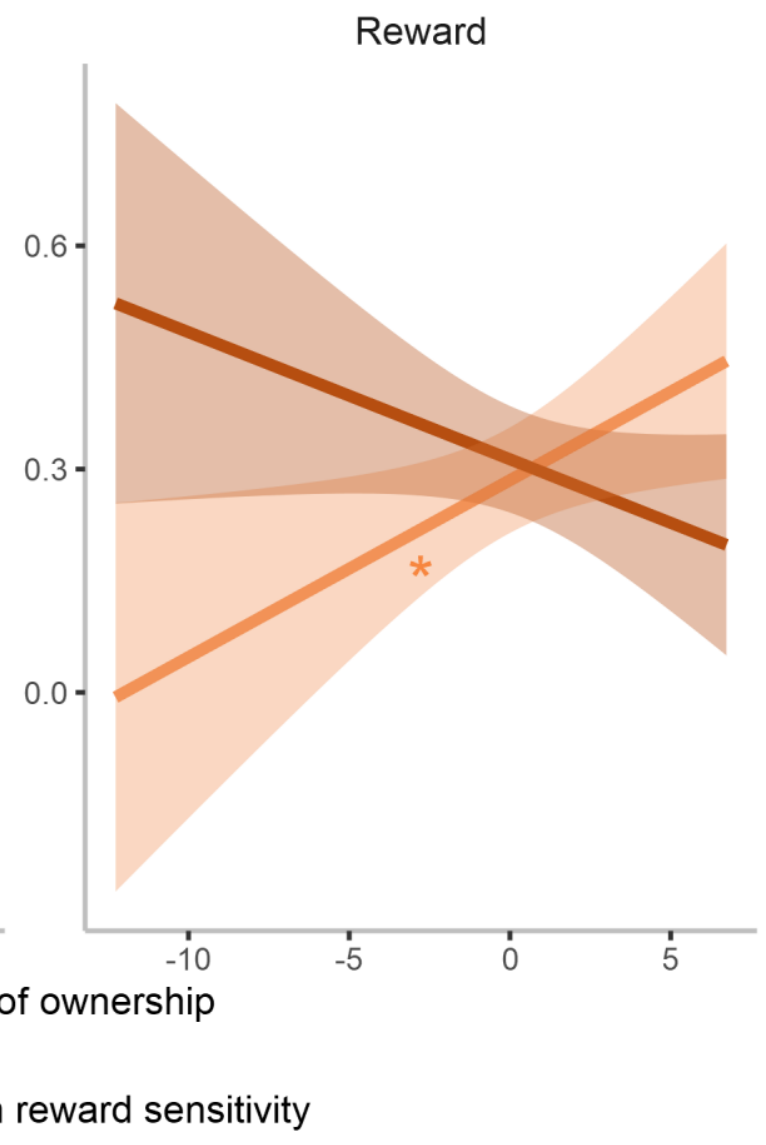

Fig.5. Lies as a function of ownership, reward sensitivity, and experimental condition.

Panel a represents lies in the no reward condition, while the reward condition is displayed in panel $\mathbf{b}$. The plot shows regression lines for 1SD below (Low Reward Sensitivity) and above (High Reward Sensitivity) the mean reward sensitivity score. The shaded bands represent 95\% confidence intervals. Asterisks indicate significance $(* p<.05)$. 